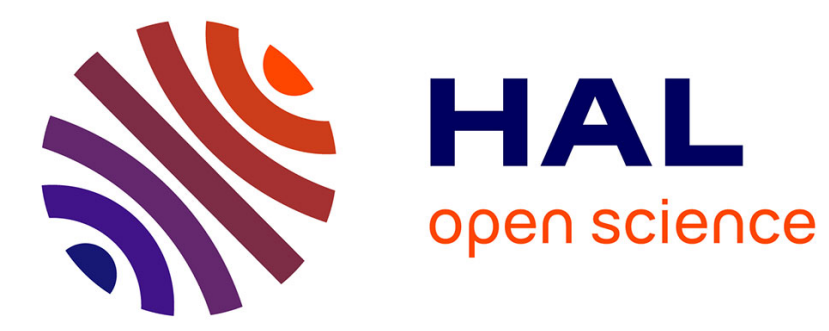

\title{
Signification physique de la lumière absorbée par la vapeur d'un métal alcalin
}

\author{
M.A. Bouchiat
}

\section{To cite this version:}

M.A. Bouchiat. Signification physique de la lumière absorbée par la vapeur d'un métal alcalin. Journal de Physique, 1965, 26 (7), pp.415-418. 10.1051/jphys:01965002607041500 . jpa-00205986

\section{HAL Id: jpa-00205986 https://hal.science/jpa-00205986}

Submitted on 1 Jan 1965

HAL is a multi-disciplinary open access archive for the deposit and dissemination of scientific research documents, whether they are published or not. The documents may come from teaching and research institutions in France or abroad, or from public or private research centers.
L'archive ouverte pluridisciplinaire HAL, est destinée au dépôt et à la diffusion de documents scientifiques de niveau recherche, publiés ou non, émanant des établissements d'enseignement et de recherche français ou étrangers, des laboratoires publics ou privés. 


\title{
SIGNIFICATION PHYSIQUE DE LA LUMIÈRE ABSORBÉE PAR LA VAPEUR D'UN MÉTAL ALCALIN
}

\author{
Par M. A. BOUCHIAT, \\ Laboratoire de Physique, École Normale Supérieure.
}

\begin{abstract}
Résumé. - L'expression générale de la lumière absorbée par unité de temps $L_{\mathrm{A}}$ est obtenue en fonction des observables décrivant le système atomique dans l'état fondamental. Dans certaines conditions d'excitation qui sont précisées, $L_{\mathrm{A}}$ peut représenter la polarisation électronique moyenne longitudinale $\left\langle S_{z}\right\rangle$, ou transversale $\left\langle S_{x}\right\rangle$, ou l'écart par rapport à l'équilibre de Boltzmann de la différence de populations entre les deux niveaux hyperfins $<\mathbf{S}$. I $>$.
\end{abstract}

\begin{abstract}
The general expression for the amount of light absorbed per unit of time $L_{\mathbf{A}}$ is given as a function of the observables which characterize an alkali metal in the ground state. It is possible to determine the characteristics of a light beam (polarization, spectral density) so that the physical meaning of $L_{\mathrm{A}}$ becomes the mean electronic longitudinal (or transversal) polarization $\left\langle S_{z}>\right.$ (or $<S_{x}>$ ) or becomes the observable $\langle\mathbf{S . I}>$ which can be expressed as the population difference of the two ground state h. f. s. levels.
\end{abstract}

De très nombreuses expériences de pompage optique utilisent comme signaux la lumière absorbée $L_{A}$, sur un faisceau lumineux détecteur traversant la vapeur orientée. $L_{\mathrm{A}}$ dépend de nombreux paramètres ; c'est en fait une combinaison linéaire (équation (1) ci-après) des éléments $\sigma \underset{\mathrm{F}}{F^{\prime} m_{F}^{\prime} \mathbf{F}^{\prime}}$ de la matrice densité atomique (les sous-niveaux de l'état fondamental sont repérés par $F m_{F}$ ) dont les coefficients (équation (2)) sont déterminés par les caractéristiques du faisceau lumineux : profil $u(k)$ de la raie excitatrice, polarisation $\mathbf{e}_{\lambda_{0}}$. En vue de rendre son interprétation physique plus directe, on peut expliciter directement $L_{\mathrm{A}}$ comme une combinaison linéaire des observables attachées au système atomique. Dans le cas d'un métal alcalin, citons parmi elles $\left\langle S_{z}\right\rangle\left(\left\langle S_{x}\right\rangle\right)$ polarisation électronique longitudinale (ou transversale), $<I_{z}>$ polarisation nucléaire, $<\mathbf{S}$. I $>$ écart par rapport à l'équilibre de Boltzmann de la différence de populations entre les deux niveaux hyperfins $F_{+}=I+1 / 2$ et $F_{-}=I-1 / 2$ distants de $\Delta W$. Avec certains choix de $u(k)$ et $\mathbf{e}_{\lambda_{\mathrm{a}}}, L_{\mathrm{A}}$ peut d'ailleurs ne dépendre que d'une seule de ces observables et avoir alors une signification particulièrement simple.

Il est clair que les résultats expérimentaux relatifs $\grave{a} L_{\mathrm{A}}$ ne seront interprétables que dans la mesure où toutes les caractéristiques du faisceau détecteur sont définies et précisées. Il n'en est malheureusement pas ainsi dans de nombreuses publications. Les conséquences de cette situation sont particulièrement graves dans les études de relaxation : la lecture du mémoire ne permet pas de dire (et en général l'auteur ne sait pas) l'observable dont la relaxation est étudiée. L'étude théorique de la relaxation d'échange entre atomes alcalins [1], ou de la relaxation par des interactions faibles de type magnétique (relaxation par collisions sur des parois ou contre les molécules d'un gaz tampon) [2] montre que les diverses observables relaxent de façon très différente et que les constantes de temps associées peuvent différer par un ordre de grandeur. En outre, si plusieurs interactions désorientatrices sont simultanément présentes, la relaxation peut être dominée par des interactions différentes pour les diverses observables (il en est ainsi pour $\left\langle S_{z}\right\rangle$ et $<\mathbf{S} . \mathbf{I}>$ lors des collisions de l'atome de $\mathrm{Rb}$ sur des parois paraffinées).

Une autre difficulté tient à l'absorption par la vapeur elle-même. Il faut que $u(k)$ et $\mathbf{e}_{\lambda_{0}}$ soient les mêmes dans tout le volume de la cellule, c'est-àdire que l'absorption soit très faible et la diffusion multiple négligeable.

Dans le but de clarifier la situation, il nous paraît important de donner l'expression générale de $L_{\mathrm{A}}$, ce qui permet en outre d'interpréter la modulation (aux fréquences Zeeman ou hyperfines) susceptible d'apparaître quand les atomes sont soumis à un champ de radiofréquence.

Les notations sont les suivantes : on illumine la vapeur par la raie $D_{1}$ (ou $\left.D_{2}\right)$. Les sous-niveaux de ${ }^{2} P_{1 / 2}\left(\right.$ ou $\left.{ }^{2} P_{3 / 2}\right)$ sont désignés par $\mathfrak{F}_{\mathscr{F}}$, les structures hyperfines par $\Delta W$. L'étude du processus "instantané » d'absorption se fait en adaptant au cas des alcalins la théorie de J. P. Barrat et C. Cohen-Tannoudji [3]. On obtient :

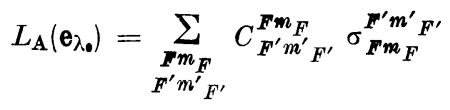

avec

$$
\begin{aligned}
& C_{\boldsymbol{F}^{\prime} m^{\prime} F^{\prime}}^{\mathbb{F}^{\prime} m_{F}}=\sum_{\mathcal{F}^{\prime} m_{\mathcal{F}}} \frac{1}{2}\left(\Gamma_{\boldsymbol{F} \mathscr{F}}^{\prime}+\Gamma_{\boldsymbol{F}^{\prime} \mathfrak{F}}^{\prime}\right) \\
& \left\langle\boldsymbol{F} m_{\boldsymbol{F}}\left|\boldsymbol{\theta}_{\lambda_{0}} . \mathbf{D}\right| \mathfrak{F} m_{\mathscr{F}}\right\rangle\left\langle\mathcal{F} m_{\mathcal{F}}\left|\boldsymbol{\theta}_{\lambda_{\boldsymbol{\theta}}}{ }^{*} \cdot \mathbf{D}\right| \boldsymbol{F}^{\prime} m_{\boldsymbol{F}^{\prime}}>\right.
\end{aligned}
$$


D est un opérateur vectoriel hermitique sans dimensions, proportionnel au moment dipolaire électrique de l'atome défini par :

$$
\begin{aligned}
& <\frac{1}{2} J_{z}^{\prime}\left|\mathbf{D}^{(0)}\right| J J_{z}>=\left(J J_{z} \mid 10 \frac{1}{2} J_{z}^{\prime}\right) \mathbf{u}^{(0)} \\
& <\frac{1}{2} J_{z}^{\prime}\left|\mathbf{D}^{( \pm 1)}\right| J J_{z}>=\mp\left(J J_{z} \mid 1 \pm 1 \frac{1}{2} J_{z}^{\prime}\right) \mathbf{u}^{( \pm 1)} .
\end{aligned}
$$

Les coefficients de Clebsch-Gordan

$$
\left(J J_{z} \mid 1 q \frac{1}{2} J_{z}^{\prime}\right)
$$

sont écrits avec les notations de Edmonds [4]. $\mathbf{u}^{(0)}, \mathbf{u}^{(+1)}$ et $\mathbf{u}^{(-1)}$ sont trois vecteurs orthogonaux définis à partir des vecteurs unitaires :

$$
\mathbf{u}^{(0)}=\mathbf{u}_{z} \quad \mathbf{u}^{( \pm 1)}=\frac{1}{\sqrt{2}}\left(\mathbf{u}_{x} \pm i \mathbf{u}_{y}\right)
$$

$\Gamma_{\mathrm{F}}^{\prime}$ est donné par :

$$
\begin{aligned}
& \Gamma_{\mathscr{F}}^{\prime}= \\
& 2 \int_{0}^{+\infty} u(k)\left|A_{k}\right|^{2} \mathrm{~d} k \frac{\Gamma / 2}{\left[k-\widetilde{k_{0}}(F \mathscr{F})+\mathbf{k}_{0}(F \mathcal{F}) \cdot \mathbf{v}\right]^{2}+\Gamma^{2} / 4}
\end{aligned}
$$

$\mathbf{v}$ étant la vitesse de l'atome, $\Gamma$ la largeur naturelle du niveau excité, $\widetilde{k}_{0}(F \mathscr{F})$ la distance énergétique entre les niveaux $F$ et $\mathcal{F}_{\text {(y compris la self énergie }}$ de $\mathcal{F}), A_{k}$ - défini comme dans [3] - traduit l'échange d'énergie entre l'atome et le champ de rayonnement.

Si $\Delta$ désigne la largeur de la raie excitatrice, la condition $\Delta \gg \Delta W$ se trouve en pratique toujours réalisée pour les alcalins $\left(\Gamma_{F \mathcal{F}}^{\prime}\right.$ devient $\Gamma_{F}^{\prime}$, indépendant de $\mathcal{F})$ : les raies $D_{1}$ et $\left(D_{2}\right)$ n'ont plus que deux composantes hyperfines d'intensité $i_{1}$ et $i_{2}$ distantes de $\Delta W$. L'excitation est dice " $n$ raie lai ge " sur l'ensemble des composantes hyperfines si l'on a de plus $\Delta \gg \Delta W: \Gamma_{F \mathcal{F}}^{\prime}$ est alors indépendantà la fois de $F$ et $\mathscr{F}$. (La condition $\Delta \ll \Delta W$ correspond au contraire au cas d'une excitation en (" raie étroite ».)

L'équation (1) est valable quel que soit le type d'excitation optique, si la matrice densité ne comporte pas d'éléments de matrice non diagonaux du type $\sigma_{F m_{F}^{\prime}}^{F^{\prime} m^{\prime}}$ avec $F \neq F^{\prime}$ (cohérences hyperfines); par contre en présence de cohérences hyperfines cette équation ne décrit les effets liés à l'existence de $\sigma_{F^{\prime} m_{F}^{\prime} F^{\prime}}^{F^{\prime}}$ avec $F \neq F^{\prime}$ (modulation à la fréquence hyperfine) que dans l'hypothèse d'une excitation optique en " raie large " [6].

L'influence de $u(k)$ est traduite par les coefficients $\Gamma^{\prime} ; \mathbf{e}_{\lambda_{0}}$ intervient par les éléments de matrice de D : la probabilité du processus d'absorption dépend des moments angulaires relatifs du système atomique dans l'état fondamental et du photon absorbé. Ceci suggère d'exprimer algébriquement un élément de matrice dipolaire électrique (entre états excité et fondamental) en fonction de ceux du moment angulaire (entre deux sous-niveaux de l'état fondamental). Par analogie avec $\mathbf{D}$, nous définissons l'opérateur vectoriel hermitique sans dimensions S par ses composantes $S^{(q)}$

$$
S^{(0)}=S_{z} \quad S^{( \pm 1)}=\frac{1}{i \cdot \overline{2}}\left(S_{x} \pm i S_{y}\right) .
$$

Les composantes de $\mathbf{D}$, de $\mathbf{S}$ et de $\mathbf{u}$ se transforment par rotation comme les harmoniques sphériques d'ordre 1 définies avec la convention de phase $\left(Y^{l m}\right)^{*}=Y^{l-n}$.

Excitation par la raie $D_{1}$ seule. - L'état excité est l'état $P_{1 / 2}$ de même moment angulaire que l'état fondamental. A chaque état propre de la fonction d'onde électronique de $S_{1 / 2}, \mid \frac{1}{2} J_{z}^{\prime}>$, correspond un état propre de la fonction d'onde électronique de $P_{1 / 2}$ ayant mêmes nombres quantiques $\mid \frac{1}{2} J_{z}^{\prime}>$. Cette correspondance est réciproque. On peut donc associer à chaque élément de matrice de $\mathbf{D}$ un (et un seul) élément de matrice de S. L'application du théorème de Wigner-Eckart aux harmoniques sphériques vectoriels permet d'écrire :

$$
\left\langle S_{1 / 2} J_{z}^{\prime}\left|\mathbf{D}^{(q)}\right| P_{1 / 2} J_{z}>=\lambda<S_{1 / 2} J_{z}^{\prime}\left|\mathbf{S}^{(q)}\right| S_{1 / 2} J_{z}>.\right.
$$

Ainsi défini, $\lambda$ est un nombre qui ne dépend pas de $q, J_{z}, J_{z}^{\prime}$. Lorsqu'on tient compte du spin nucléaire $I$, la correspondance subsiste encore entre un état $\mid \mathscr{F}_{\mathscr{F}^{\prime}}>$ de $P_{1 / 2}$ et un état $\mid F^{\prime \prime} m^{\prime \prime}{ }_{r^{\prime \prime}}>$ de $S_{1 / 2}$ de la manière suivante : ce sont tous les deux des états $\mid L_{1 / 2} I \alpha>$ (pour $\mid F m_{k^{\prime}}>$, état $S$; pour $\mid \mathfrak{F} m_{\mathscr{F}}>$, état $P$ ) représentés par la même combinaison linéaire a (caractérisée par les coefficients $C_{J_{z} I_{z}}^{\alpha}$ ) des fonctions d'onde électronique et nucléaire.

$$
\left.\left|L_{1 / 2} I \alpha>=\sum_{J z I z} C_{J_{\iota} \boldsymbol{I}_{z} \mid L_{1 / 2}}^{\alpha} J_{z}>\right| I I_{z}\right\rangle .
$$

Les opérateurs $\mathbf{D}$ et $\mathbf{S}$ n'agissant que sur la partie électronique de la fonction d'onde, la relation (3) devient :

$$
\left\langle P_{1 / 2} I \alpha^{\prime}\left|\mathbf{D}^{(\alpha)}\right| S_{1 / 2} I \alpha>=\lambda<S_{1 / 2} I \alpha^{\prime}\left|\mathbf{S}^{(q)}\right| S_{1 / 2} I \alpha>\right.\text {. }
$$

En explicitant les deux membres, on trouve $\lambda^{2}=4 / 3$.

Par ailleurs, l'ensemble des états $\left|S_{1 / 2} F m_{H}\right\rangle$ constitue pour un opérateur $S^{(q)}$, n ensemble complet d'états propres.

En tenant compte des remarques précédentes, 
l'expression de $L_{\mathrm{A}}$ dans le cas d'une excitation $D_{1}$ devient :

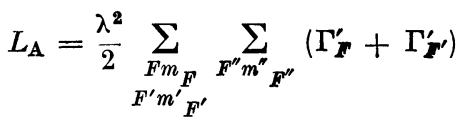

$$
\begin{aligned}
& <F m_{F}\left|\boldsymbol{\theta}_{\lambda_{0}} . \mathrm{S}\right| F^{\prime \prime} m^{\prime \prime}{ }_{F^{\prime \prime}}><F^{\prime \prime} m^{\prime \prime}{ }_{F^{\prime \prime}}\left|\mathrm{e}_{\lambda_{0}}^{*} . S\right| F^{\prime} m_{F^{\prime}}^{\prime}>\sigma_{F m_{F}}^{F^{\prime} m_{F^{\prime}}^{\prime}}
\end{aligned}
$$

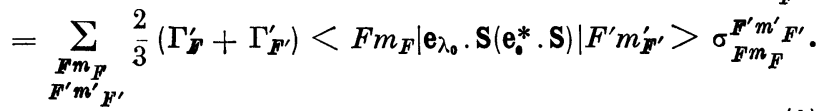

Comme $S=1 / 2$ l'opérateur $\mathrm{e}_{\lambda_{0}} . S\left(\mathrm{e}_{\lambda_{0}}^{*} . S\right)$ peut s'écrire sous une forme linéaire en $S^{(\boldsymbol{(})}$ :

$$
e_{\lambda_{0}} . S\left(e_{\lambda_{0}}^{*} . S\right)=\frac{1}{2}\left(e_{\lambda_{0}} \wedge e_{\lambda_{0}}^{*}\right) \cdot S+\frac{1}{4} \theta_{\lambda_{0}} \cdot e_{\lambda_{0}}^{*}
$$

Excitation par la raie $D_{2}$ seule. - Le théorème de Wigner-Eckart ne permet plus d'évaluer la sommation sur les états $P_{3 / 2} \mathscr{F} m_{\mathcal{F} \text {. Pour trans- }}$ former l'expression de $L_{\mathrm{A}}$, on utilise la relation suivante :

$$
\begin{aligned}
& \sum_{\mathscr{F} m_{\mathscr{F}}}\left\langle F m_{F}\left|\boldsymbol{e}_{\lambda_{0}} . \mathbf{D}\right| P_{1 / 2} \mathscr{F} m_{\mathscr{F}}\right\rangle\left\langle P_{1 / 2} \mathscr{F} m m_{F}\left|\mathbf{e}_{\lambda_{0}}^{*} \cdot \mathbf{D}\right| F^{\prime} m_{\boldsymbol{F}^{\prime}}^{\prime}\right\rangle
\end{aligned}
$$

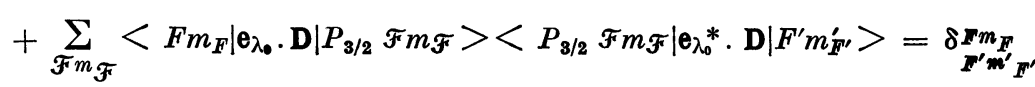

Elle se démontre à partir d'une relation de fermeture portant sur l'ensemble des états excités $\mathcal{F} m_{\mathscr{F}}\left(\right.$ de $P_{1 / 2}$ et de $P_{3 / 2}$ ) et à partir des équations de définition de $D^{(a)}$. Nous obtenons :

$$
\begin{aligned}
& L_{\mathbf{A}}=\sum_{\substack{F m^{\prime} \\
F^{\prime} m^{\prime} T^{\prime \prime}}} \frac{1}{2}\left(\Gamma_{\boldsymbol{F}}^{\prime}+\Gamma_{\boldsymbol{F}^{\prime}}^{\prime \prime}\right) \\
& {\left[1-\frac{4}{3}<F m_{F}\left|\Theta_{\lambda_{0}} . S\left(\mathbf{e}_{\lambda_{0}}^{*} . S\right)\right| F^{\prime} m_{F^{\prime}}^{\prime}>\sigma_{F m_{F}^{\prime}}^{W^{\prime} m^{\prime}}\right] .}
\end{aligned}
$$

Les équations (4) et (5) conduisent aux remarques suivantes :

$1^{0} \mathrm{Si}$ l'excitation optique se fait simultanément par $D_{1}$ et par $D_{2}$ avec égale intensité $L_{\mathrm{A}}$ est totalement indépendante de l'état du système atomique.

Ce cas se produit en particulier lorsque la largeur de la raie excitatrice est grande devant l'écart de structure fine de l'état excité $\Delta S$. L'interprétation physique de ce résultat est la suivante :

Dans l'état excité, le moment magnétique porté par $\mathbf{L}$ se couple à $\mathbf{S}$ en un temps de l'ordre de $1 / \Delta S$; dans l'état fondamental par contre $L=0$, et ce couplage n'existe pas. Au cours de la transition dipolaire électrique seule la partie orbitale de la fonction d'onde interagit directement avec le champ de rayonnement. Cohen-Tannoudji a montré que l'on peut interpréter $1 / \Delta$ comme étant « la durée effective " du processus d'absorption (3). Si $1 / \Delta$ est court devant $1 / \Delta S, \mathbf{S}$ ne précesse pratiquement pas autour de $\mathbf{L}$ pendant la durée du processus d'absorption et celui-ci est donc totalement indépendant de l'état angulaire de $\mathbf{S}$.

$2^{\circ}$ En employant un filtre interférentiel, il est possible pour certains alcalins, de n'utiliser que la raie $D_{1}$ (ou la raie $D_{2}$ ) pour l'excitation. L'examen de l'expression (4) (ou (5)) montre que la probabilité du processus d'absorption dépend alors de l'état angulaire électronique du système atomique dans l'état fondamental. Cependant deux cas sont encore à envisager : a) Si l'excitation se fait avec des composantes hyperfines d'égale intensité $\left(i_{1}=i_{2}\right)$, en posant $\Gamma_{F}^{\prime}=1 / T_{p}$, l'expression (4) conduit pour $D_{1}$ à :

$$
L_{\mathrm{A}}=\frac{4}{3 T_{p}} \operatorname{Tr}\left\{\mathbf{e}_{\lambda_{0}} . \mathbf{S}\left(\mathbf{e}_{\lambda_{0}^{*}}^{*} . \mathbf{S}\right) \sigma\right\}
$$

Il est clair que la probabilité pour qu'un photon $\mathbf{e}_{\lambda_{0}}$ soit absorbé ne dépend que de l'état angulaire ÉLEGTRONIQUE $d u$ système atomique dans l'état fondamental. C'est en particulier le cas d'une excitation en "raie large »; le résultat s'interprète alors physiquement par un raisonnement analogue à celui développé plus haut: $1 / \Delta W$ (et $1 / \Delta W$ ) représentent les temps nécessaires pour que $\mathbf{J}$ et $\mathbf{I}$ se couplent magnétiquement dans l'état fondamental (et excité). Ces temps sont à comparer à la " durée du processus d'absorption ", 1/ $\Delta$. Dans le cas où $\Delta \gg \Delta W, \Delta W$ le noyau et l'électron n'ont pas le temps de se coupler pendant la durée du processus d'absorption, ni dans l'état initial, ni dans l'état final : la probabilité du processus d'absorption ne peut pas dépendre de l'état angulaire nucléaire de l'état fondamental.

En particulier, si le faisceau détecteur est longitudinal et polarisé en $\sigma^{+}$dans la direction d'excitation $\left(\mathrm{e}_{\lambda_{0}} \cdot \mathbf{S}=S^{(-1)}\right)$, on trouve le résultat que nous avions déjà énoncé et utilisé antérieurement (5) :

$$
\begin{aligned}
& L_{\mathrm{A}}=\frac{2}{3 T_{p}}<\frac{1}{2}-S_{z}>\quad\left(\text { pour } D_{1}\right) \\
& L_{\mathrm{A}}=\frac{2}{3 T_{p}}<1+S_{z}>\quad\left(\text { pour } D_{2}\right) .
\end{aligned}
$$

En réalisant la condition $i_{1}=i_{2}$ dans la source émettrice, on peut étudier l'évolution de l'observable $\left\langle S_{z}\right\rangle$ sur $L_{A}$. D'autre part, les expressions ci-dessus permettent de prévoir que si l'excitation est en " raie large » $L_{A}$ sera sensible à l'existence de cohérences hyperfines $\sigma_{F m_{F}}^{F^{\prime} m^{\prime} F^{\prime}}$ et de calculer les modulations correspondantes. 
Si le faisceau détecteur est transversal (parallèle à $\mathrm{Ox}$ ) et de polarisation $\sigma^{+}$par rapport à $\mathrm{Ox}, L_{\boldsymbol{A}}$ permet de détecter le moment électronique transversal :

$$
\begin{aligned}
& L_{\mathrm{A}}=\frac{2}{3 T_{p}}<\frac{1}{2}-S_{x}>\quad\left(\text { pour } D_{1}\right) \\
& L_{\mathrm{A}}=\frac{2}{3 T_{p}}<1+S_{\infty}>\quad\left(\text { pour } D_{2}\right) .
\end{aligned}
$$

Remarque. - Toutes les relations utilisées jusqu'ici ne font pas l'hypothèse que $F$ ou $\mathcal{F}$ sont de bons nombres quantiques et les résultats obtenus sont valables pour une excitation en " raie large " pour l'ensemble des composantes Zeeman et hyperfines de $D_{1}$ (ou $D_{2}$ ) et pour des valeurs du champ statique $H_{0}$ pouvant atteindre des milliers de gauss (valeur à laquelle l'effet Zeeman est encore petit devant l'écart de structure fine $\Delta S$ de l'état excité).

b) Dans le cas où l'excitation optique est au contraire en "raie étroite ", le processus d'absorption peut dépendre de l'état angulaire NUCLÉAIRE de l'état fondamental. Dans un but de simplification d'écriture, on suppose pour la suite que l'excitation se fait en champ faible (écart Zeeman petit devant $\Delta W$ $\operatorname{avec} i_{1} \neq i_{2}$. En se limitant au cas où il n'existe pas de cohérences hyperfines $\sigma_{F m_{F}}^{{F^{\prime}}^{\prime}{ }^{\prime}}$ on a (pour $D_{1}$ ):

$$
\begin{aligned}
L_{\mathbf{A}} & =\frac{2}{3}\left(\Gamma_{F+}^{\prime}+\Gamma_{F-}^{\prime}\right) \operatorname{Tr}\left\{\mathbf{e}_{\lambda_{0}} \cdot \mathbf{S}\left(\mathbf{e}_{\lambda_{0}^{*}}^{*} \cdot \mathbf{S}\right) \sigma\right\} \\
& +\frac{2}{3}\left(\Gamma_{F+}^{\prime}-\Gamma_{F-}^{\prime}\right) \operatorname{Tr}\left\{\mathbf{e}_{\lambda_{0}} \cdot \mathbf{S}\left(\mathbf{e}_{\lambda_{0}^{*}}^{*} \cdot \mathbf{S}\right)\left(\sigma_{F+}-\sigma_{F-}\right)\right\}
\end{aligned}
$$

$L_{\mathrm{A}}$ n'est plus également sensible à l'état angulaire électronique des deux niveaux $F_{+}$et $F$ - (représentés respectivement par les matrices densités $\sigma_{F+}$ et $\sigma_{F_{-}}$) et dépend de la différence de populations entre $F_{+}$et $F_{-}$.

En particulier, si le faisceau détecteur n'est pas polarisé, on obtient quelle que soit sa direction :

$$
\begin{aligned}
L_{\mathbf{A}}=\frac{1}{6}\left(\Gamma_{F+}^{\prime}+\Gamma_{F-}^{\prime}\right)+\frac{1}{6}\left(\Gamma_{\boldsymbol{F}+}^{\prime}-\Gamma_{F-}^{\prime}\right) \\
\operatorname{Tr}\left(\sigma_{F+}-\sigma_{\boldsymbol{F}-}\right)=L_{0}+K<\mathbf{S . I}>
\end{aligned}
$$

$L_{0}$ est la lumière absorbée par la vapeur à l'équilibre de Boltzmann. $K$ est proportionnel à $i_{2}-i_{1}$. $L_{\mathbf{A}}$ permet l'étude de l'observable $<\mathbf{S}$. I $>$ [5],[6].

Dans le cas où le faisceau longitudinal est polarisé $\sigma_{+}$, l'interprétation du signal, donné par (7), devient complexe : elle fait intervenir $\operatorname{Tr}\left(\sigma_{\mathrm{F}+}-\sigma_{\mathrm{F}-}\right), \operatorname{Tr}\left\{S_{z} \sigma_{\mathrm{F}+}\right\}$ et $\operatorname{Tr}\left\{S_{z} \sigma_{\mathrm{F}-}\right\}$ ou encore les observables $<\mathbf{S}$.I $>$. $\left\langle S_{z}>\right.$ et $\left\langle I_{z}\right\rangle$. On comprend donc que ce cas soit déconseillé expérimentalement. Nous avons indiqué [5] un procédé expérimental permettant de déterminer si l'on se trouve dans les cas $a$ ) ou $b$ ).

Nous résumons dans le tableau ci-dessous les principaux résultats donnant l'expression de $L_{\mathrm{A}}$ pour certaines conditions d'excitation $\left(u(k), \mathbf{e}_{\lambda_{0}}\right)$ en fonction de l'intensité lumineuse $\left(1 / T_{\mathfrak{p}}\right)$ et des diverses observables caractérisant le système atomique dans l'état fondamental. Ces résultats sont déduits de la formule générale (1) en supposant $\Delta \gg \Delta w$. La définition précise des termes en " raie large » et en "raie étroite » est donnée dans le texte.

\section{Polarisation du faisceau lumineux}

Profil SPectral

$$
\text { - }
$$

$D_{1}$ et $D_{2}$ " raie large *

$D_{1}$ " raie large "

$D_{2}$ " raie large "

$D_{1}$ (ou $D_{2}$ ) " raie étroite " ( $H_{0}$ faible) $\sigma \pm$ PAR RAPPORT A Oz

$\frac{\overline{1}}{T_{\mathrm{p}}}$

$$
\frac{2}{3 T_{\mathrm{p}}}\left(\frac{1}{2} \mp<S_{z}>\right)
$$$$
\frac{2}{3 T_{\mathrm{p}}}\left(1 \pm<S_{\mathrm{z}}>\right)
$$

combinaison linéaire de

$$
\left\{\begin{array}{c}
<\mathbf{S . I}> \\
\operatorname{Tr}\left\{\sigma_{F+} S_{z}\right\} \\
\operatorname{Tr}\left\{\sigma_{F-S} S_{z}\right.
\end{array}\right\}
$$

$\sigma \pm$ PAR RAPPORT A $\mathrm{O}_{x}$

$\overline{1}$

$\frac{2}{3 T_{\mathrm{p}}}\left(\frac{1}{2} \mp<S_{\mathrm{x}}>\right)$

$\frac{2}{3 T_{\mathrm{p}}}\left(1 \pm<S_{\mathrm{x}}>\right)$

combinaison linéaire de

$$
\left\{\begin{array}{c}
<\mathbf{S . I}> \\
\operatorname{Tr}\left\{\sigma_{\boldsymbol{F}}+S_{\boldsymbol{x}}\right\} \\
\operatorname{Tr}\left\{\sigma_{\boldsymbol{F}} S_{\boldsymbol{x}}\right\}
\end{array}\right.
$$

LUMIÈRE NATURELLE DIREGTION QUELCONQUE

$$
\begin{gathered}
\frac{1}{T_{\mathrm{p}}} \\
\frac{1}{3 T_{\mathrm{p}}} \\
\frac{2}{3 T_{\mathrm{p}}}
\end{gathered}
$$

$$
L_{0}+K<\text { S.I }>
$$

Manuscrit reçu le 20 mai 1965.

\section{BIBLIOGRAPHIE}

[1] Grossetete (F.), J. Physique, 1965, 25, 383.

[2] Bouchiat (M. A.), J. Physique, 1963, 24, 379 et 611.

[3] Barrat (J. P.) et Cohen-Tannoudji (C.), J. Physique Rad., 1961, 22, 329 et 443.

Cohen-Tannoudji (C.), Thèse, Paris, 1962 ; Annales Physique, 1962, 7, 443 et 469 .
[4] Edmonds (A. R.), Angular momentum in quantum electronics (chap. 7), Princeton University Press, Princeton, 1957.

[5] Bouchiat (M. A.) et Brosser (J.), C. R. Acad. Sc., $1962,254,3650$ et 3828 et $1963,257,2825$.

[6] Bouchint (M. A.), Thèse, Paris, 1964. 